11 Hofmeyr GJ, Nikodem VC, de Jager M, Drakely A. Side-effects of oral misoprostol in the third stage of labour-a randomised placebocontrolled trial. S Afr Med J 2001;91:432-5.

12 Lumbiganon P, Villar J, Piaggio G, Gulmezoglu AM, Adetoro L, Carroli G. Side effects of oral misoprostol during the first 24 hours after administration in the third stage of labour. BJOG 2002;109: $1222-6$

13 Bamigboye AA, Hofmeyr GJ, Merrell DA. Rectal misoprostol in the prevention of postpartum hemorrhage: a placebo-controlled trial. Am J Obstet Gynecol 1998;179:1043-6.

14 Hofmeyr GJ, Nikodem VC, de Jager M, Gelbart BR. A randomised placebo controlled trial of oral misoprostol in the third stage of labour. $B r$ Obstet Gynaecol 1998;105:971-5.

15 Surbek DV, Fehr PM, Hosli I, Holzgreve W. Oral misoprostol for third stage of labor: a randomized placebo-controlled trial. Obstet Gynecol 1999;94:255-8.

16 Prasertcharoensuk W, Swadpanich U, Lumbiganon P. Accuracy of the blood loss estimation in the third stage of labor. Int J Gynaecol Obstet 2000;71:69-70.

17 Hoj L, da Silva D, Hedegaard K, Sandstrom A, Aaby P. Factors associated with maternal mortality in rural Guinea-Bissau. A longitudinal population-based study. BJOG 2002;109:792-9.

\title{
Cluster randomised trial of intermittent preventive treatment for malaria in infants in area of high, seasonal transmission in Ghana
}

\author{
Daniel Chandramohan, Seth Owusu-Agyei, Ilona Carneiro, Timothy Awine, \\ Kwame Amponsa-Achiano, Nathan Mensah, Shabbar Jaffar, Rita Baiden, Abraham Hodgson, \\ Fred Binka, Brian Greenwood
}

\begin{abstract}
Objective To evaluate the effects of intermittent preventive treatment for malaria in infants (IPTi) with sulfadoxine-pyrimethamine in an area of intense, seasonal transmission.

Design Cluster randomised placebo controlled trial, with 96 clusters allocated randomly to sulfadoxine-pyrimethamine or placebo in blocks of eight.

Interventions Children received

sulfadoxine-pyrimethamine or placebo and one month of iron supplementation when they received DPT-2, DPT-3, or measles vaccinations and at 12 months of age.
\end{abstract}

Main outcome measures Incidence of malaria and of anaemia determined through passive case detection. Results $89 \%(1103 / 1242)$ of children in the placebo group and 88\% (1088/1243) in the IPTi group completed follow-up to 24 months of age. The protective efficacy of IPTi against all episodes of malaria was $24.8 \%$ (95\% confidence interval $14.3 \%$ to $34.0 \%$ ) up to 15 months of age. IPTi had no protective effect against malaria between 16 and 24 months of age (protective efficacy $-4.9 \%,-21.3 \%$ to $9.3 \%)$. The incidence of high parasite density malaria $(\geq 5000$ parasites $/ \mu \mathrm{l})$ was higher in the IPTi group than in the placebo group between 16 and 24 months of age (protective efficacy $-19.5 \%,-39.8 \%$ to $-2.2 \%)$. IPTi reduced hospital admissions with anaemia by $35.1 \%$ (10.5\% to $52.9 \%)$ up to 15 months of age. IPTi had no significant effect on anaemia between 16 and 24 months of age (protective efficacy $-6.4 \%,-76.8 \%$ to $35.9 \%)$. The relative risk of death up to 15 months of age in the IPTi group was 1.26 (95\% confidence interval 0.81 to $1.96 ; \mathrm{P}=0.31$ ), and from 16 to 24 months it was 1.28 (0.77 to 2.14; $\mathrm{P}=0.35$ ).

Conclusions Intermittent preventive treatment for malaria with sulfadoxine-pyrimethamine can reduce malaria and anaemia in infants even in seasonal, high transmission areas, but concern exists about possible rebound in the incidence of malaria in the second year of life.

\section{Introduction}

Malaria remains the leading cause of morbidity and mortality in young children in impoverished communities in Africa. It is estimated that 515 (range 300-600) million clinical attacks of falciparum malaria occurred globally in 2002 and that $70 \%$ of these cases occurred in Africa. ${ }^{1}$ Malaria is one of the major causes of anaemia in children in sub-Saharan Africa, accounting for an estimated $18 \%$ of the disability adjusted life years lost because of anaemia. ${ }^{2}$ The number of deaths attributable to malaria associated anaemia is estimated at 190000 to 974000 a year. ${ }^{3}$ In high intensity transmission areas, the highest burden of malaria and malaria associated anaemia is in infants. ${ }^{4}$

Control of malaria and anaemia depends largely on passive case detection and appropriate treatment. However, many children who have malaria parasitaemia, anaemia, or both remain asymptomatic, ${ }^{5}$ and chronic asymptomatic parasitaemia increases the risk of severe anaemia. ${ }^{6}$ Chemoprophylaxis during the first year of life significantly reduced the incidence of malaria and anaemia in a study in Tanzania. ${ }^{7}$ The risk of malaria and anaemia increased in the second year of life after chemoprophylaxis had been stopped, however, suggesting that complete protection against malaria infection during infancy had impaired naturally acquired immunity against the infection. Two recent studies in Tanzania, done in areas with perennial transmission of malaria, showed that administration of a full therapeutic course of an antimalarial at set times during infancy, regardless of whether or not the infant was parasitaemic (intermittent preventive treatment in infants (IPTi)), substantially reduced the incidence of malaria and anaemia. ${ }^{89}$ The antimalarial was given in

A figure and three tables are on bmj.com
London School of Hygiene and Tropical Medicine, London

WC1E 7HT

Daniel

Chandramohan

clinical senior lecturer

Ilona Carneiro

lecturer

Shabbar Jaffar senior lecturer

Brian Greenwood director, Gates Malaria Partnership

Kintampo Health Research Centre, Kintampo, Ghana Seth Owusu-Agyei director

Navrongo Health Research Centre, Navrongo, Ghana Timothy Awine research officer Kwame Amponsa-Achiano research physician Nathan Mensah data manager Rita Baiden research physician Abraham Hodgson director

INDEPTH-Network Accra, Ghana

Fred Binka executive director

Correspondence to: D Chandramohan daniel.chandramohan@ lshtm.ac.uk

BMJ 2005;331:727-33 
association with vaccination in one study and at the time of growth monitoring visits in the other. Whether IPTi linked to a child immunisation schedule will have a similar protective effect in areas with intense, seasonal transmission is not known. We aimed to evaluate the effects of IPTi with sulfadoxinepyrimethamine in an area of intense, seasonal transmission in northern Ghana.

\section{Methods}

Study site

We did the study in the Kassena-Nankana district in the Upper East region of Ghana, which lies within the open woodland, sub-Sahel region of West Africa. Annual rainfall averages $850 \mathrm{~mm}$, with most rain falling during June to October. The entomological inoculation rate was estimated to be 418 infective bites per person per year in 2001-2, and almost all infective bites occur between June and November, coinciding with the rainy season..$^{10}$ The prevalence of parasitaemia in 2-12 month old infants at the end of the dry season in June 2000 was $8.6 \%$ (95\% confidence interval 6.6\% to $11.0 \%)$; this increased to $52.8 \%(48.7 \%$ to $56.7 \%)$ at the end of the rainy season (November 2000). The prevalence of a packed cell volume $<24 \%$ showed a similar seasonal pattern, increasing from $2.4 \%(1.4 \%$ to $3.9 \%)$ in the dry season to $12.6 \%(10.1 \%$ to $15.4 \%)$ at the end of the rainy season (unpublished data from baseline simple random sample cross sectional surveys). The 14 day adequate clinical and parasitological response rate after treatment with sulfadoxinepyrimethamine for clinical malaria (geometric mean parasite density $18978 / \mu \mathrm{l}$, range $2000-530000 / \mu \mathrm{l}$ ) in children aged $6-59$ months was $77.6 \%(68.9 \%$ to $84.8 \%$ ) in 2004. ${ }^{11}$ However, the day 14 parasite clearance rate in asymptomatic children aged 19-45 months (geometric mean parasite density $2595 / \mu \mathrm{l}$, range $120-48640 / \mu \mathrm{l})$ was $94.5 \%(90.2 \%$ to $97.3 \%)$ (unpublished data from drug sensitivity study carried out in the IPTi study children in 2004, after all children had completed follow-up at 18 months of age).

A demographic surveillance system for monitoring vital events has been operating in this district since 1993. The area is divided into four zones, and within each zone households (compounds) are grouped into clusters. On average, a cluster has 100 households. The district contained 244 clusters of households in 2000. We restricted the study to the central and southern zones, which had a total of 96 clusters of households. All infants living in these clusters of households without a history of allergy to sulfadoxinepyrimethamine were eligible for enrolment in the study. The study area has one hospital with 140 beds and 11 community health centres that provide outpatient care only. In addition, there are 18 outreach clinics where only expanded programme of immunisation (EPI) and growth monitoring are provided. The study infants were enrolled in these 30 EPI delivery points.

\section{Primary objective and sample size}

The primary objective was to determine the effect of IPTi on the incidence of anaemia. We assumed that the incidence of anaemia would be $25 \%$ in the placebo group and $18.7 \%$ in the IPTi group and that the coeffi- cient of variation between clusters $(\mathrm{k})$ would be 0.1 . With the available 96 clusters, each of 25 children on average, the study had approximately $95 \%$ power to detect a statistically significant difference in the incidence of anaemia at the $5 \%$ two sided significance level.

\section{Randomisation and study drugs}

We randomly allocated 96 clusters of households to sulfadoxine-pyrimethamine or placebo in blocks of eight clusters by using a computer generated list. To increase blinding, we assigned clusters allocated to sulfadoxine-pyrimethamine or placebo to eight different drug codes (four sulfadoxine-pyrimethamine and four placebo). A statistician at the London School of Hygiene and Tropical Medicine generated and kept safe the randomisation list of clusters and drug codes. The study team and caretakers of study children were blinded to the drug codes. Study infants received half a tablet of sulfadoxine-pyrimethamine or an identical placebo when they received DPT-2 or DPT-3 vaccines and one tablet of sulfadoxine-pyrimethamine or placebo when they received measles vaccine and at 12 months of age, according to the cluster where they lived. In addition to sulfadoxine-pyrimethamine or placebo, all infants received one month's supply of iron supplement $(2.5 \mathrm{ml}, 15 \mathrm{mg}$ elemental iron, twice weekly for four weeks) when they received DPT-2, DPT-3, or measles vaccines and at 12 months of age. We obtained sulfadoxine-pyrimethamine and placebo from Cosmos Pharmaceuticals, Nairobi. The content and solubility of the sulfadoxine-pyrimethamine tablets were confirmed by solubility testing and high performance liquid chromatography at the London School of Hygiene and Tropical Medicine. Study drugs were crushed and mixed with water and administered by a study field worker at an EPI clinic.

\section{Enrolment and follow-up}

Enrolment of study infants started in September 2000, and follow-up to 24 months of age was completed in June 2004. The nature of the trial was explained to caretakers of infants attending the study EPI clinics for DPT-1 vaccine, and they were invited to enrol their infant in the study. After informed written consent had been obtained, infants were enrolled, assigned a unique personal identity number, and allocated to the respective study group according to the cluster where they lived. All study infants were given a photo identity card, and their guardians were asked to bring the card whenever they visited an EPI clinic or other health facility. A field worker visited the households of study infants one or two days before the date when DPT-2 (IPT-1), DPT-3 (IPT-2), and measles (IPT-3) vaccinations were due to remind caretakers to attend an EPI clinic. A similar visit was made shortly before the IPT-4 administration at the age of 12 months was due. Finger prick blood samples for assessing packed cell volume, malaria parasitaemia, and the immune response to EPI vaccines were taken when infants received IPT-1, IPT-3, and IPT-4. Immune responses to EPI vaccines are being tested as part of a larger study commissioned by the IPTi consortium, and these results will be reported elsewhere.

We used a morbidity questionnaire to assess all study children who presented with any illness at one of the 11 health centres or at the hospital outpatient 
department. We collected blood samples for malaria parasites from all children who had fever or a history of fever during the previous two days. We used an inpatient morbidity questionnaire to assess children who were admitted to the hospital and took blood samples for microscopy and determination of packed cell volume.

Study children were visited at home at 18 months of age to collect finger prick blood samples for assessing packed cell volume and the presence of malaria parasitaemia and again at 23 months of age to assess their health. The study physician certified the cause of death of children who died in the hospital. We assessed cause of death of children who died at home by verbal autopsy. ${ }^{12}$

A fieldworker visited a random 20\% sample of study children at home within four weeks after administration of IPT dose 1 or dose 2 to assess adherence to administration of iron at home and to inquire about adverse events. Children who had any rash other than scabies, heat rash, or insect bites were referred to the project physician for a clinical assessment.

\section{Ethical clearance and analytical plan}

An independent statistician (Simon Cousens) and epidemiologist (Laura Rodrigues) approved the analytical plan. We cleaned and audited the data before locking the data and unmasking the intervention codes. The primary outcomes, defined in the analytical plan, were the incidence of all episodes of malaria associated fevers (history of fever or temperature $\geq 37.5^{\circ} \mathrm{C}$ plus malaria parasites detected on a blood smear) detected in study children attending the health centres or the hospital and the incidence of anaemia (packed cell volume $<24 \%$ ) in children admitted to the study hospital for any illness. We stratified analyses by age (2-15 months and 16-23 months) to assess the effect of IPTi during the intermittent treatment phase (2-15 months) and after the effect of the intervention had ceased (16-23 months). We decided a priori to investigate the effect of sex, urban residence, and mosquito net use on the outcome, including only those covariates that were significantly associated with the outcome $(\mathrm{P}<0.1)$ in the final model.

Because of the highly seasonal nature of transmission of malaria at the study site, we compared the effect of IPTi administered during the wet season (JulyNovember) with IPTi administered during the dry season (December-June). We restricted this analysis to 2-12 month old infants. We estimated the protective efficacy of IPTi against malaria, anaemia, and hospital admissions by all combinations of timing of IPTi doses.

\section{Statistical methods}

We analysed data on an intention to treat basis, including all infants who were enrolled, at the time of receipt of DPT-1, regardless of whether they received any doses of IPT. Time at risk started at enrolment, approximately four weeks before the first dose was administered and will, therefore, give a conservative estimate of the effect of IPTi. Thirty episodes of malaria occurred in 127.5 person years at risk in the placebo group and 30 episodes in 127.1 person years at risk in the sulfadoxine-pyrimethamine group in the period between enrolment and the first dose received. We analysed data by using random effects Poisson or negative binomial regression models to allow for intra- cluster correlation at the level of randomisation (household cluster) and to adjust for important covariates. We used Poisson regression to analyse the incidence of anaemia, cerebral malaria, hospital admissions, and death. We used negative binomial regression to analyse the incidence of malaria associated fevers and high density fevers, as these were significantly clustered within individuals. We calculated person time at risk from the date of enrolment to second birthday, date of migration, or date of death. In addition, if a child had received an antimalarial drug during the follow-up period, we subtracted the 28 day posttreatment period from the person time at risk. We analysed data on cross sectional outcomes by using a random effects logistic regression model.

\section{Results}

Between September 2000 and June 2004, 2485 infants were enrolled in the study and followed up until the age of 24 months, death, or migration out of the study area. The number of children per cluster varied but was well balanced between the two groups. It ranged from 2 to 47 (mean 30.1) in the IPTi group and from 7 to 47 (mean 29.5) in the placebo group. The proportions of infants who received drug treatment at different time points and the proportion of children followed up to 24 months of age were similar between the IPTi and control groups (see figure on bmj.com). Table A on bmj.com shows the characteristics of the infants in each arm of the study. Tables 1 and 2 summarise the effects of IPTi on malaria, anaemia, and other outcomes.

\section{Incidence of malaria}

The incidence of malaria was significantly lower in the IPTi group than in the placebo group up to 15 months of age (table 1). The protective efficacy of IPTi against all episodes of malaria was 24.8\% (95\% confidence interval $14.3 \%$ to $34.0 \%$ ). IPTi had no effect on the incidence of malaria between 16 and 24 months of age (protective efficacy $-4.9 \%,-21.3 \%$ to $9.3 \%$ ). From 2 to 24 months of age IPTi had a protective efficacy of $16.1 \%$ (6.4\% to $24.8 \%$ ) (table 2). Kaplan-Meier survival analysis of time to first episode of malaria (figure) suggests that much of the protective effect against malaria occurred after the first and second doses, with some indication of waning of effect with time (log rank $\chi^{2}=16.4, \mathrm{P}=0.0001$ for $0-8$ months; $\log \operatorname{rank} \chi^{2}=8.86$, $\mathrm{P}=0.0029$ for $9-15$ months, and $\log$ rank $\chi^{2}=0.14$, $\mathrm{P}=0.7093$ for 16 -24 months). Although the protective efficacy against all malaria episodes during a one month post-IPTi period was not significantly different between the four courses of IPTi, during the second month after IPTi the protective efficacy of IPTi dose 4 was significantly lower than that seen after dose 2 (table 3).

Protective efficacy of IPTi against malaria attacks associated with high parasite density (defined a priori as $\geq 5000$ parasite/ $\mu \mathrm{l}$ ) up to 15 months of age was similar to the protective efficacy against malaria attacks of any parasite density $(23.3 \%, 10.8 \%$ to $34.1 \%)$ (table 2). The incidence of high parasite density malaria was significantly higher in the IPTi group than in the placebo group between 16 and 24 months of age (protective efficacy $-19.5 \%,-39.8 \%$ to $-2.2 \%$ ). From 
Table 1 Outcomes in sulfadoxine-pyrimethamine (SP) and placebo arms by age group

\begin{tabular}{|c|c|c|c|c|c|c|c|c|c|c|}
\hline \multirow[b]{3}{*}{ Outcomes } & \multicolumn{5}{|c|}{ 2-15 months } & \multicolumn{5}{|c|}{ 16-24 months } \\
\hline & \multicolumn{2}{|c|}{ Placebo } & \multicolumn{2}{|c|}{ SP } & \multirow{2}{*}{$\begin{array}{l}\text { Odds ratio } \\
\text { (95\% CI) }\end{array}$} & \multicolumn{2}{|c|}{ Placebo } & \multicolumn{2}{|c|}{ SP } & \multirow{2}{*}{$\begin{array}{l}\text { Odds ratio } \\
(95 \% \mathrm{CI})\end{array}$} \\
\hline & Events & Rate $^{*}$ & Events & Rate* & & Events & Rate* & Events & Rate* & \\
\hline \multicolumn{11}{|l|}{ Malaria } \\
\hline Person years at risk & - & 1325 & - & 1324 & - & - & 732 & - & 722 & - \\
\hline All episodes of malaria & 1348 & 1017.0 & 1009 & 762.2 & 0.75 (0.66 to 0.86$) \dagger$ & 579 & 790.8 & 609 & 843.9 & 1.05 (0.91 to 1.21$) \ddagger$ \\
\hline Malaria ( $\geq 5000$ parasites/ $\mu$ l) & 813 & 613.4 & 627 & 473.6 & 0.76 (0.66 to 0.89 ) & 337 & 460.3 & 403 & 558.4 & 1.19 (1.02 to 1.39$)$ \\
\hline \multicolumn{11}{|l|}{ Hospital admissions } \\
\hline Person years at risk & - & 1418 & - & 1415 & - & - & 763 & - & 756 & - \\
\hline All causes & 457 & 322.3 & 403 & 284.8 & 0.87 (0.73 to 1.05$)$ & 139 & 182.2 & 136 & 179.8 & $1.0(0.98$ to 1.31$)$ \\
\hline All malaria admissions & 85 & 60.7 & 53 & 37.5 & 0.60 (0.42 to 0.86$)$ & 35 & 45.9 & 32 & 42.3 & 0.92 (0.54 to 1.56$)$ \\
\hline$P C V<24 \%$ & 97 & 68.4 & 63 & 44.5 & $0.65(0.47$ to 0.89$) \S$ & 35 & 45.9 & 37 & 48.9 & 1.09 (0.66 to 1.81)ף \\
\hline $\mathrm{PCV}<15 \%$ & 18 & 12.7 & 10 & 7.1 & 0.56 (0.26 to 1.21$)$ & 7 & 9.2 & 4 & 5.3 & 0.57 (0.16 to 2.04 ) \\
\hline Malaria + PCV<24\% & 49 & 34.6 & 23 & 16.3 & $0.47(0.28$ to 0.77$)$ & 11 & 14.4 & 14 & 18.5 & 1.28 (0.56 to 2.95$)$ \\
\hline Malaria + PCV $<15 \%$ & 8 & 5.6 & 5 & 3.5 & 0.64 (0.21 to 1.95$)$ & 4 & 5.2 & 0 & 0 & - \\
\hline Cerebral malaria & 21 & 14.8 & 15 & 10.6 & 0.68 (0.35 to 1.35$)$ & 16 & 21.0 & 12 & 15.9 & 0.81 (0.33 to 1.99 ) \\
\hline \multicolumn{11}{|l|}{ Deaths } \\
\hline Person years at risk & - & 1418 & - & 1415 & & - & 763 & - & 756 & - \\
\hline All causes & 35 & 24.6 & 44 & 31.1 & 1.27 (0.82 to 1.99$)$ & 26 & 34.1 & 33 & 43.7 & 1.27 (0.76 to 2.13$)$ \\
\hline Malaria or anaemia & 20 & 14.1 & 24 & 17.0 & 1.21 (0.66 to 2.23$)$ & 12 & 15.7 & 14 & 18.5 & 1.17 (0.54 to 2.53$)$ \\
\hline
\end{tabular}

$\mathrm{PCV}=$ packed cell volume

*Per 1000 child years at risk.

+Intracluster correlation log rank test $\chi^{2}=34.04 ; P<0.001$

Intracluster correlation log rank test $\chi^{2}=4.77 ; P=0.014$

§Intracluster correlation log rank test $\chi^{2}=0.03 ; \mathrm{P}=0.435$.

IIntracluster correlation log rank test $\chi^{2}=1.26 ; \mathrm{P}=0.131$.

2 to 24 months of age IPTi had a protective efficacy of $11.5 \%(0.0 \%$ to $21.6 \%)$ against high parasite density malaria.

\section{Incidence of anaemia}

The incidence of all episodes of anaemia (packed cell volume $<24 \%$ ), identified through passive case detection, was significantly lower in the IPTi group than in the placebo group up to 15 months of age (table 1). The protective efficacy of IPTi against anaemia was $35.1 \%$ (10.5\% to $52.9 \%)$. IPTi had no significant effect on the incidence of anaemia between 16 and 24 months of age (protective efficacy $-6.4 \%,-76.8 \%$ to $35.9 \%$ ). From 2 to 24 months of age IPTi had a protective efficacy of $23.8 \%(-1.3 \%$ to $42.6 \%)$ against anaemia (table 2).

The incidence of anaemia associated with malaria parasitaemia was also significantly lower in the IPTi group up to 15 months of age (table 1). The protective efficacy of IPTi against malaria associated anaemia was
$53 \%(22.8 \%$ to $71.3 \%)$. The incidence of malaria associated anaemia increased in the IPTi group by $26.3 \%$ ( $-44.9 \%$ to $189.8 \%$ ) between 16 and 24 months of age, but this difference was not statistically significant. From 2 to 24 months of age IPTi had a protective efficacy of $10.5 \%(-1.6 \%$ to $19.3 \%)$ against malaria associated anaemia (table 2 ).

\section{Incidence of severe disease and mortality}

The incidence of hospital admissions for all causes was $13.2 \%$ ( $-4.5 \%$ to $28.0 \%)$ lower in the IPTi group than in the placebo group up to 15 months of age, but this reduction was not statistically significant (table 2). We found no significant difference between groups in the all cause hospital admission rate between 16 and 24 months. The incidence of all cause hospital admissions from 2 to 24 months of age was slightly lower in the IPTi group, but this difference was not statistically significant $(9.5 \%,-7.5 \%$ to $23.8 \%)$.

Table 2 Protective efficacy (95\% confidence interval) and number of children protected per 1000 ( $95 \%$ confidence interval), by age group

2-15 months

16-24 months

2-24 months

\begin{tabular}{|c|c|c|c|c|c|c|}
\hline \multirow[b]{2}{*}{ Outcome } & \multicolumn{2}{|c|}{ 2-15 months } & \multicolumn{2}{|c|}{$16-24$ months } & \multicolumn{2}{|c|}{ 2-24 months } \\
\hline & Protective efficacy (\%)* & No protected/1000 & Protective efficacy (\%)* & No protected/1000 & Protective efficacy $(\%)^{*}$ & No protected/1000 \\
\hline \multicolumn{7}{|l|}{ Malaria } \\
\hline All episodes of malaria & 24.9 (14.5 to 34.1 ) & 254.8 (183.0 to 326.6 ) & -4.9 ( -21.3 to 9.3$)$ & $-53.0(-146.0$ to 39.9$)$ & 16.3 (6.7 to 25.0$)$ & $\begin{array}{c}145.5 \\
\text { (88.7 to 202.4) }\end{array}$ \\
\hline Malaria $\geq 5000 / \mu \mathrm{l}$ & 23.6 (11.1 to 34.3 ) & 139.7 (83.6 to 195.9 ) & $-18.9(-39.1$ to -1.7$)$ & $-98.1(-171.5$ to -24.7$)$ & $11.9(0.5$ to 22.0$)$ & 55.4 (10.8 to 100.0$)$ \\
\hline \multicolumn{7}{|l|}{ Hospital admissions } \\
\hline All causes & 12.7 (-4.8 to 27.3$)$ & 37.5 (3.1 to 78.1 ) & -0.4 (-31.3 to 23.3$)$ & 2.4 (-40.4 to 45.2$)$ & 9.5 (-7.5 to 23.8$)$ & 25.1 (5.3 to 55.4$)$ \\
\hline All malaria admissions & 39.8 (14.0 to 57.9 ) & 22.5 (6.2 to 38.7 ) & 8.4 (-56.0 to 46.2$)$ & 3.6 (-17.5 to 24.7$)$ & 31.2 (5.4 to 49.9 ) & 15.9 (3.0 to 28.8 ) \\
\hline $\mathrm{PCV}<24 \%$ & 35.5 (11.2 to 53.1) & 23.9 (6.4 to 41.4$)$ & -9.0 (-81.3 to 34.4$)$ & -3.0 (-24.9 to 18.8$)$ & $24.2(-0.5$ to 42.8$)$ & 14.5 (0.8 to 28.2) \\
\hline PCV $<15 \%$ & 44.4 ( -20.6 to 74.3$)$ & 5.6 (1.7 to 12.9) & $43.3(-104.5$ to 84.3$)$ & $3.9(-4.7$ to 12.4$)$ & $44.1 \quad(-11.7$ to 72.1$)$ & 5.0 (0.6 to 10.6$)$ \\
\hline Malaria + PCV $<24 \%$ & 53.3 (23.4 to 71.6 ) & 18.3 (6.6 to 30.0$)$ & $-28.4(-195.1$ to 44.1$)$ & $-4.1(-17.0$ to 8.8$)$ & 38.8 (5.5 to 60.4$)$ & 10.5 (1.6 to 19.3) \\
\hline Malaria + PCV $<15 \%$ & 36.3 (-94.9 to 79.2$)$ & $2.1(-2.9$ to 7.1$)$ & - & $5.2(0.1$ to 10.4$)$ & $57.1 \quad(-25.9$ to 85.4$)$ & 3.2 (0.5 to 6.9$)$ \\
\hline Cerebral malaria & 31.6 (-35.3 to 65.4$)$ & $4.2(-4.1$ to 12.5$)$ & 18.6 (-99.2 to 66.7) & $5.1(-8.5$ to 18.8$)$ & 26.6 (-28.6 to 58.1) & 4.5 (2.7 to 11.7) \\
\hline \multicolumn{7}{|l|}{ Deaths } \\
\hline All causes & $-27.4(-98.7$ to 18.3$)$ & $-6.4(-18.7$ to 5.9$)$ & $-27.3(-112.9$ to 23.9$)$ & $-9.5(-29.4$ to 10.3$)$ & $-27.2(-78.1$ to 9.1$)$ & $-7.5(-18.1$ to 3.1$)$ \\
\hline Malaria & $-21.4(-123.2$ to 34.0$)$ & $-2.9(-12.0$ to 6.3$)$ & $-17.0(-153.1$ to 45.9$)$ & $-2.8(-15.9$ to 10.4$)$ & -19.1 (-92.0 to 25.1) & -2.8 (-10.4 to 4.7$)$ \\
\hline
\end{tabular}

$\mathrm{PCV}=$ packed cell volume

${ }^{*}$ Adjusted for sex and rural/urban residence; protective efficacy (\% reduction in outcomes in IPTi group)=(1-odds ratio)×100 


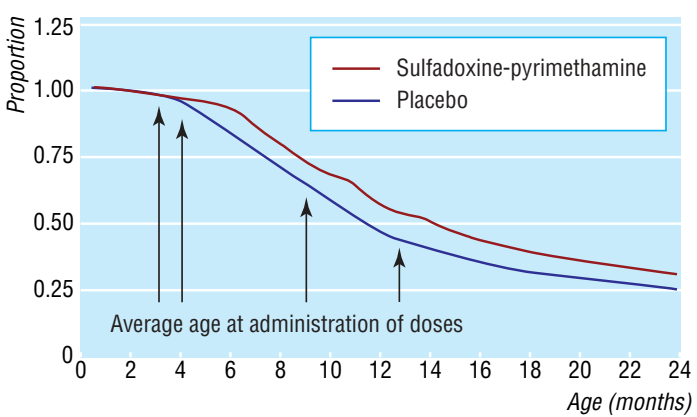

Kaplan-Meier survival estimates of time to first episode of malaria by intervention group

A significant reduction in the incidence of hospital admissions for malaria $(37.8 \%, 11.8 \%$ to $56.1 \%)$ occurred in the IPTi group up to 15 months of age. IPTi had no significant effect on hospital admissions for malaria between 16 and 24 months. From 2 to 24 months of age IPTi had a significant protective effect of $15.9 \%(3.0 \%$ to $28.8 \%)$ against hospital admissions for malaria.

More deaths occurred in the IPTi group (77) than in the placebo group (61), but this difference was not statistically significant (table 1 ). The relative risk of death up to 15 months of age in the IPTi group was $1.26(0.81$ to $1.96 ; \mathrm{P}=0.31)$, and from 16 to 24 months it was $1.28(0.77$ to $2.14 ; \mathrm{P}=0.35)$. There were 32 deaths attributable to malaria in the placebo group and 38 deaths in the IPTi group. This difference was not statistically significant.

\section{Prevalence of anaemia and parasitaemia}

Table B on bmj.com shows the prevalences of anaemia and parasitaemia at approximately 3, 9, 12, and 18 months of age. The mean packed cell volume was significantly higher in the IPTi group than in the control group at 12 months of age, but it was slightly lower in the IPTi group at 18 months. The prevalence of malaria parasitaemia was lower in the IPTi group at 9, 12 , and 18 months of age.

Effect of malaria transmission season on protective efficacy of IPTi

Table 4 shows the protective efficacy of IPTi by number of doses administered during wet and dry seasons in 2-12 month old infants. Children who received IPTi doses 1 and 2 in the wet season and dose 3 in the dry season had the maximum protective effect against malaria $(52 \%)$ and anaemia $(72 \%)$. The next best protective efficacy against malaria $(37 \%)$ was obtained by giving IPTi dose 2 during the wet season and doses 1 and 3 in the dry season. Although the effect was not statistically significant, infants who received all three
IPTi doses in the dry season also seemed to have some protection against malaria (24\%).

\section{Incidence of adverse events}

The proportions of children who vomited after administration of drugs was similar between the two groups $(0.4 \%$ in the placebo group versus $0.3 \%$ in the sulfadoxine-pyrimethamine group). Among the children who were visited at home within four weeks after administration of IPTi dose $1(\mathrm{n}=1765,74 \%)$ or dose $2(n=214,9 \%), 32(3.3 \%)$ children in the placebo group and $27(2.7 \%)$ in the sulfadoxinepyrimethamine group had skin rashes. None of the skin rashes was severe or suggestive of a drug sensitivity reaction. The incidence of outpatient attendance for any illness and hospital admissions due to any severe illness during the month after IPTi doses 1, 2, 3, and 4 was lower in the IPTi group than in the placebo group (table C on bmj.com). However, during the one month period after IPTi dose 1 , four deaths occurred in the sulfadoxine-pyrimethamine group compared with none in the placebo group. Similarly, during the month after IPTi dose 2, six deaths occurred in the sulfadoxine-pyrimethamine group compared with none in the placebo group. Causes of these deaths were as follows: malaria (4), diarrhoea disease (1), septicaemia (1), "spirit child" (probably herbal poisoning) (2), and unknown (2). None of these deaths was considered to be associated with the IPTi drugs by the panel of physicians who reviewed the verbal autopsy questionnaires.

\section{Discussion}

\section{Effect of malaria transmission intensity on IPTi efficacy}

The protective efficacy of intermittent preventive treatment with sulfadoxine-pyrimethamine against malaria and anaemia in infants observed in our study is moderate and substantially lower than that reported from Ifakara, Tanzania. ${ }^{8}$ This may be because of the differences in the intensity and pattern of malaria transmission between the two sites. The intensity of transmission is low (entomological inoculation rate $31 /$ year) and perennial in Ifakara, ${ }^{13}$ whereas it is very high (418/year) and highly seasonal in Navrongo, our study site. ${ }^{10}$ The incidence of malaria in the placebo group was 0.43 episode/child/year in Ifakara compared with 1.0 episode/child/year in Navrongo. Although the protective efficacy of IPTi was higher in Ifakara than in Navrongo, the number of episodes of malaria prevented was similar at the two sites $(260 \mathrm{v}$ 255 per 1000 children per year) because the background incidence of malaria was higher in Navrongo. In spite of a higher incidence of malaria, the

Table 3 Protective efficacy against malaria during the first and second month after each dose of IPTi

\begin{tabular}{|c|c|c|c|c|c|c|c|c|c|c|}
\hline \multirow{3}{*}{$\begin{array}{l}\text { IPTi dose } \\
\text { No }\end{array}$} & \multicolumn{4}{|c|}{$\begin{array}{l}\text { Malaria episodes during } \\
\text { first month after IPTi }\end{array}$} & \multicolumn{4}{|c|}{$\begin{array}{l}\text { Malaria episodes during } \\
\text { second month after IPTi }\end{array}$} & \multirow{3}{*}{$\begin{array}{l}\text { Protective efficacy (\%) } \\
\text { during first month after } \\
\text { IPTi }(95 \% \mathrm{CI})\end{array}$} & \multirow{3}{*}{$\begin{array}{l}\text { Protective efficacy (\% } \\
\text { during second month } \\
\text { after IPTi ( }(95 \% \mathrm{CI})\end{array}$} \\
\hline & \multicolumn{2}{|c|}{ Placebo } & \multicolumn{2}{|c|}{ SP } & \multicolumn{2}{|c|}{ Placebo } & \multicolumn{2}{|c|}{ SP } & & \\
\hline & Events & PYAR & Events & PYAR & Events & PYAR & Events & PYAR & & \\
\hline 1 & 41 & 88.4 & 10 & 90.2 & - & - & - & - & 77 (50 to 89 ) & - \\
\hline 2 & 67 & 91.8 & 11 & 92.1 & 84 & 90.8 & 48 & 90.7 & 84 (69 to 91) & 43 (19 to 60$)$ \\
\hline 3 & 119 & 82.8 & 25 & 85.2 & 125 & 81.9 & 95 & 84.4 & 80 (68 to 87 ) & 27 (4 to 44) \\
\hline 4 & 108 & 85.3 & 24 & 86.7 & 90 & 86.1 & 101 & 87.3 & 78 (65 to 86 ) & $-11(-49$ to 18$)$ \\
\hline
\end{tabular}

IPTi=intermittent preventive treatment for malaria in infants; PYAR=person years at risk; $\mathrm{SP}=$ sulfadoxine-pyrimethamine. 
Table 4 Protective efficacy of IPTi by season of administration in 2-12 month old infants. Values are percentages (95\% confidence intervals)

\begin{tabular}{|c|c|c|c|c|c|}
\hline Doses administered & $\begin{array}{l}\text { No of } \\
\text { children }\end{array}$ & $\begin{array}{l}\text { All episodes } \\
\text { of malaria** }\end{array}$ & $\begin{array}{l}\text { Malaria episodes with } \\
\text { parasite } \geq 5000 / \mu l^{*}\end{array}$ & $\begin{array}{l}\text { Anaemia } \\
(\mathrm{PCV}<24)\end{array}$ & $\begin{array}{l}\text { Hospital admissions } \\
\text { for malaria }\end{array}$ \\
\hline 1,2 , and 3 in dry season & 524 & 24 (-7 to 46$)$ & 31 (-6 to 55) & 44 (-122 to 86$)$ & 61 ( -91 to 7$)$ \\
\hline 1 in wet, 2 and 3 in dry & 363 & $2(-54$ to 37$)$ & -13 (-103 to 38$)$ & 67 (-216 to 97) & - \\
\hline 2 in wet, 1 and 3 in dry & 366 & 37 (10 to 56$)$ & 24 (-22 to 52$)$ & 17 (-232 to 79$)$ & -105 (-958 to 60$)$ \\
\hline 3 in wet, 1 and 2 in dry & 1901 & 15 (-1 to 28$)$ & 17 (-2 to 33$)$ & 29 (-31 to 62) & $43(-5$ to 69$)$ \\
\hline 1 and 2 in wet, 3 in dry & 965 & 52 (37 to 63) & 57 (38 to 70) & 72 (12 to 91$)$ & 57 (-135 to 92) \\
\hline 1 and 3,2 and 3 , or all three in wet & 112 & $28(-40$ to 63$)$ & 24 (-73 to 67) & $28(-1046$ to 4$)$ & - \\
\hline 1,2, and 3 in any season & 2098 & 27 (16 to 37$)$ & 28 (14 to 39) & 43 (11 to 64$)$ & 44 (8 to 66$)$ \\
\hline
\end{tabular}

incidences of anaemia and hospital admissions in the placebo group were lower in Navrongo than in Ifakara (anaemia: $0.07 v 0.11 /$ child/year; hospital admissions: $0.32 v 0.63 /$ child/year). This difference may be due to the fact that the Navrongo study covered a large area (some children lived up to $40 \mathrm{~km}$ from the hospital) whereas the study population of Ifakara lived within a $5 \mathrm{~km}$ radius of the hospital. The incidences of anaemia and hospital admissions are probably underestimated in both the placebo and IPTi groups in our study, and thus the public health impact of IPTi on these outcomes may also be underestimated; more cases may have been prevented than we were able to detect.

\section{Effect of malaria transmission season on IPTi efficacy}

IPTi dose 2 seemed to have a higher protective efficacy against malaria than IPTi doses 1 and 3. Children who received their IPTi dose 2 in the wet season had 46\% protection against malaria between 2 and 12 months of age, whereas those who received this dose in the dry season had only $16 \%$ protection. Similarly, children who received dose 1 in the wet season had $42 \%$ protection compared with $19 \%$ protection in those who received dose 1 in the dry season. Children who received dose 3 in the wet season had only 15\% protection compared with $38 \%$ protection in those who had dose 3 in the dry season. This is because children who received dose 3 in the dry season had received dose 1 , dose 2 , or both in the wet season. Children who received IPTi doses 1 and 2 in the wet season and dose 3 in the dry season had the maximum protection against malaria (52\%), which is comparable to the protective efficacy of IPTi observed in Ifakara (62\%). It seems that offering IPTi with sulfadoxinepyrimethamine at the time of DPT-2 and DPT-3 during the wet season alone would have the maximum benefit, although giving IPTi year round may be beneficial in this area with high seasonal transmission. However, as this study was not designed to detect the efficacy of different dosing schedules, these data need to be interpreted with caution. In Senegal, Cisse et al observed $86 \%$ protection against clinical attacks of malaria by administering three doses of sulfadoxinepyrimethamine plus artesunate to children under 5 years old on three occasions during the malaria transmission season, ${ }^{14}$ and similar results have been obtained in Mali. ${ }^{15}$ Further studies of appropriate dosing schedules for IPTi in high, seasonal transmission areas are needed.

\section{Mode of action of IPTi}

Protection against malaria during the one month period after an IPTi dose is much higher than the overall effect of IPTi from 2 to 15 months of age. This suggests that IPTi using sulfadoxine-pyrimethamine reduces the incidence of malaria and anaemia, at least in part, through its chemoprophylactic effect, which is known to last for about a month. However, the fact that doses 1 and 2 administered during the high transmission season had a higher protective efficacy than dose 3 suggests that reducing the number of infections between 3 and 6 months of age may modulate the immune response and thereby give greater protection for prolonged periods. This hypothesis needs to be tested by using long acting and short acting antimalarials and studying their effect on immune responses.

\section{Rebound effect of IPTi}

The IPTi study from Tanzania did not find a rebound increase in the incidence of malaria when IPTi was stopped and even found some evidence of persistence of protection into the second year of life.$^{16}$ However, we found a significant increase in malaria attacks associated with high density parasitaemia and a slight decrease in mean packed cell volume between 16 and 24 months of age when the effect of IPTi had worn off. This could be due to the differences in the level of intensity of transmission. Our finding of a potential rebound effect suggests that a careful monitoring of rebound effect is needed if IPTi is implemented on a large scale. Sulfadoxine-pyrimethamine IPTi did not increase the incidence of common adverse events such as vomiting and skin rash, and the incidence of any illness that needed medical attention was lower in the IPTi group than in the placebo group. However, 10 deaths occurred during the first month after IPTi whereas none occurred in the placebo group in this period. Although none of the deaths was deemed to be caused by IPTi, this trend needs to be carefully monitored in all IPTi studies and implementation programmes.

\section{Effect of insecticide treated nets plus IPTi}

Insecticide treated bed nets reduce the incidence of malaria and anaemia substantially. A meta-analysis showed that nets reduce episodes of malaria by $50 \%$ (95\% confidence interval $45 \%$ to $55 \%$ ) and increase mean packed cell volume by $1.7 \%$ in children aged under 5 years. ${ }^{17}$ A combination of nets plus malaria chemoprophylaxis (pyrimethamine-dapsone) reduced the incidence of malaria by $97 \%(92 \%$ to $99 \%)$ and increased the mean packed cell volume by $1.2 \%$ 


\section{What is already known on this topic}

Intermittent preventive treatment for malaria in infants with sulfadoxine-pyrimethamine substantially reduced the incidence of malaria and anaemia in Tanzania

This was in an area of low, perennial transmission of malaria

\section{What this study adds}

Four courses of sulfadoxine-pyrimethamine given at DPT-2, DPT-3, and measles vaccination and at 12 months of age reduced malaria (25\%) and anaemia (35\%) up to age 15 months in a high, seasonal transmission area in Ghana

The incidence of high parasite density malaria increased 20\% when treatment was stopped

compared with nets alone. ${ }^{18}$ Only $17 \%$ of the placebo group and 19\% of the IPTi group in our study population had used any form of net. Among the children who used a net, addition of IPTi gave $19 \%$ ( $-2 \%$ to $35 \% ; \mathrm{P}=0.07)$ protection against malaria compared with children who received the placebo. Those using a net had no rebound effect of high parasite density malaria between 16 and 24 months of age (protective efficacy $4 \%,-47 \%$ to $38 \% ; \mathrm{P}=0.83$ ). These results should be interpreted with caution, however, as this study was not designed to evaluate the combined effect of nets plus IPTi. Distribution of nets is increasingly linked with immunisation, and the coverage of nets in children attending immunisation clinics has increased remarkably from $4 \%$ to $94 \%$ in a district in Ghana. ${ }^{19}$ If this approach to the delivery of nets continues, both nets and IPTi will reach the same population, so assessing the combined effect of nets and IPTi on malaria is important, particularly the possible rebound effect in the second year of life. Any potential rebound effect might be offset by the continued protection offered by nets. On the other hand, the net plus IPTi combination might give almost total protection against malaria infection during infancy and impair naturally acquired immunity, as seen with continuous chemoprophylaxis, ${ }^{7}$ and increase the burden of severe malaria later in life. ${ }^{20}$

\section{Conclusions}

More data are needed to decide on the appropriate dose schedule for IPTi in areas with high seasonal transmission and its interaction with insecticide treated bed nets. However, intermittent preventive treatment in infants with sulfadoxine-pyrimethamine linked to the expanded programme of immunisation schedule has the potential to reduce the burden of malaria even in areas with high, seasonal transmission.

We thank the Kassena-Nankana district health management team, the project staff, and the study participants for their kind cooperation and support. We also thank Harparkash Kaur for testing the contents and quality of the study drugs.

Contributors: DC was involved in protocol development, implementation of the trial, data analysis, and drafting the manuscript; he is the guarantor. SO-A and FB were involved in protocol development, implementation of the trial, and reviewing the manuscript. IC was involved in data analysis and drafting the manuscript. TA and NM were involved in implementation of the trial, data management, and reviewing the manuscript. KA-A, $\mathrm{RB}$, and $\mathrm{AH}$ were involved in implementation of the trial and reviewing the manuscript. SJ was involved in randomisation, data analysis, and reviewing the manuscript. BG was involved in protocol development, monitoring of the trial, data analysis, and drafting the manuscript.

Funding: This study was funded by a grant from the Department for International Development (DFID) UK (grant No R7602). The analytical plan and the manuscript were not influenced by the DFID. The DFID cannot accept any responsibility for the information and views presented in this manuscript. The subsidiary study of sensitivity of malaria parasites to sulfadoxine-pyrimethamine was funded by the Gates Malaria Partnership.

Competing interest statement: None declared.

Ethical approval: The study was approved by the ethical committees of the Ministry of Health of Ghana and the London School of Hygiene and Tropical Medicine.

1 Snow R, Guerra C, Noor A, Myint H, Hay S. The global distribution of clinical episodes of Plasmodium falciparum malaria. Nature 2005;434:214-7.

2 Murray CJ, Lopez AD. Global burden of disease and injury series. Geneva: World Health Organization, 1996:320-7.

3 Murphy SC, Breman JG. Gaps in the childhood malaria burden in Africa: cerebral malaria, neurological sequelae, anaemia, respiratory distress, hypoglycaemia and complications of pregnancy. Am J Trop Med Hyg 2001;64(1-2 suppl):57-67.

4 Schellenberg D, Menendez C, Kahigwa E, Font F, Galindom C, Acosta C, et al. African children with malaria in an area of intense Plasmodium falciparum transmission: features on admission to the hospital and risk factors for death. Am J Trop Med Hyg 1999;61:431-8.

5 Schellenberg D, Schellenberg JR, Mushi A, Savigny D, Mgalula L, Mbuya C, et al. The silent burden of anaemia in Tanzanian children: a community-based study. Bull World Health Organ 2003;81:581-90.

6 Kitua AY, Smith TA, Alonso PL, Urassa H, Masanja H, Kimario J, et al. The role of low level Plasmodium falciparum parasitaemia in anaemia among infants living in an area of intense and perennial transmission. Trop Med Int Health 1997;2:325-33.

7 Menendez C, Kahigwa E, Hirt R, Vounatsou P, Aponte JJ, Font F, et al. Randomised placebo-controlled trial of iron supplementation and malaria chemoprophylaxis for prevention of severe anaemia and malaria in Tanzanian infants. Lancet 1997;350:844-50.

8 Schellenberg D, Menendez C, Kahigwa E, Aponte J, Vidal J, Tanner M, et al. Intermittent treatment for malaria and anaemia control at time of routine vaccinations in Tanzanian infants: a randomised, placebocontrolled trial. Lancet 2001;357:1471-7.

9 Massaga JJ, Kitua AY, Lemnge MM, Akida JA, Malle LN, Ronn AM, et al. Effect of intermittent treatment with amodiaquine on anaemia and malarial fevers in infants in Tanzania: a randomised placebo-controlled trial. Lancet 2003;361:1853-60.

10 Appawu M, Owusu-Agyei S, Dadzie S, Asoala V, Anto F, Koram K, et al. Malaria transmission dynamics at a site in northern Ghana proposed for testing malaria vaccines. Trop Med Int Health 2004;9:164-70.

11 Oduro AR, Anyorigiya T, Hodgson A, Ansah P, Anto F, Ansah NA, et al. A randomized comparative study of chloroquine, amodiaquine and randomized comparative study of chloroquine, amodiaquine and
sulphadoxine-pyrimethamine for the treatment of uncomplicated sulphadoxine-pyrimethamine for the treatment of
malaria in Ghana. Trop Med Int Health 2005;10:279-84.

12 Population, Health and Survival at Indepth sites. Ottawa, Canada: International Development Research Centre, 2002.

13 Drakeley C, Schellenberg D, Kihonda J, Sousa CA, Arez AP, Lopes D, et al. An estimation of the entomological inoculation rate for Ifakara: a semiurban area in a region of intense malaria transmission in Tanzania. Trop Med Int Health 2003;8:767-74.

14 Cisse B, Sokhna CS, Alexander N, Trape JF, Greenwood B. A double blind, randomised placebo-controlled trial to measure the potential of SIPT with artesunate pus SP to reduce the malaria burden in Niakhar, Senegal. Am J Trop Med Hyg 2004;71(suppl):abstract 17.

15 Dicko A, Sagara I, Sissoko M, Guindo O, Diallo A, Kone M, et al. Impact of intermittent preventive treatment with sulphadoxine-pyrimethamine targeting the transmission season on the incidence of clinical malaria in children of 6 months to 10 years in Kambili, Mali. Am J Trop Med Hyg 2004;71(suppl):abstract 18.

16 Schellenberg D, Menendez C, Aponte J, Kahigwa E, Tanner M, Mshinda $\mathrm{H}$, et al. Intermittent treatment antimalarial treatment for Tanzanian infants: follow-up to age 2 years of a randomised, placebo-controlled infants: follow-up to age 2 year
trial. Lancet $2005 ; 365: 1481-3$.

17 Lengeler C. Insecticide-treated bed nets and curtains for preventing malaria. Cochrane Database Syst Rev 2004;(2):CD000363.

18 Greenwood BM, Greenwood AM, Bradley AK, Snow RW, Byass P, Hayes $\mathrm{RJ}$, et al. Comparison of two strategies for control of malaria within a primary health care programme in the Gambia. Lancet 1988;i:1121-7.

19 Grabowsky M, Nobiya T, Ahun M, Donna R, Lengor M, Zimmerman D, et al. Distributing insecticide-treated bednets during measles vaccination: a low-cost means of achieving high and equitable coverage. Bull World Health Organ 2005;83:195-201.

20 Snow RW, Marsh K. The consequences of reducing transmission of Plasmodium falciparum in Africa. Adv Parasitol 2002;52:235-64. 